\title{
EQUILIBRIUM POINTS OF NONATOMIC GAMES OVER A BANACH SPACE ${ }^{1}$
}

\author{
BY
}

M. ALI KHAN

\begin{abstract}
Schmeidler's results on the equilibrium points of nonatomic games with strategy sets in Euclidean $n$-space are generalized to nonatomic games with strategy sets in a Banach space. Our results also extend previous work of the author which assumed the Banach space to be separable and its dual to possess the Radon-Nikodym property. Our proofs use recent results in functional analysis.
\end{abstract}

1. Introduction. In this paper we study Cournot-Nash equilibria of games with a continuum of players, each of whom has a strategy set in a Banach space. The importance of such games for economic theory has been recently underscored by Dubey, Mas-Colell and Shubik [7]; see their Example 2, in particular. However, [7] is primarily concerned with the equivalence of Walrasian and Cournot-Nash equilibria. Our focus here is on the existence theory.

The results reported here can be seen as a continuation of research initiated by Schmeidler [19], who formulated and studied nonatomic games over $n$-dimensional Euclidean space. They also extend earlier work of the author in at least three important respects. To begin with, [14] assumed that each player's strategy set lies in a separable Banach space whose dual has the Radon-Nikodým property. This condition is not satisfied for the infinite-dimensional spaces typically studied in economics; see Bewley [2] and Mas-Colell [18]. It is of interest that such an assumption can be relaxed at the cost of a rather mild uniformity assumption on the distribution of strategy sets. Secondly, it seems desirable to have results for conjugate Banach spaces which are not separable and for which the strategy sets are not weakly compact but only norm bounded and weak* closed. We also present such results here. They are especially relevant for commodity spaces studied in [2 and 18]. Finally, unlike [14], our results on the existence of mixed-strategy equilibria rely only on preference orderings rather than on payoff functions. It is not known to us whether our conditions on preferences are sufficient for them to be represented as jointly measurable payoff functions. Such theorems are difficult to obtain even for a finite-dimensional setting; see Wesley [21].

Received by the editors September 27, 1983.

1980 Mathematics Subject Classification. Primary 90A14, 28C20, 46N05.

Key words and phrases. Nonatomic game, Gel'fand integral, Diestel's theorem, approximate pure strategy equilibrium.

${ }^{1}$ A preliminary version of this paper was presented at the N.S.F.-N.B.E.R. Conference on Mathematical Economics held in April 1983 at Yale University. 
The proofs of our theorems use recent results in functional analysis, specifically the theory of vector measures. In particular, our proofs lean heavily on results due to Castaing and Valadier [3], Diestel [4], Leese (see Theorem 8.4 in [20]) and Rosenthal (see Theorem 13, p. 252 in [5]).

$\S 2$ of the paper develops the necessary preliminaries, and $\S 3$ is devoted to the model and results. $\$ 4$ presents the proofs.

2. Notational and terminology. Let $(T, \mathscr{T}, \mu)$ be a complete, finite, nonatomic measure space; i.e., $\mu$ is a real-valued, nonnegative, countably additive, nonatomic measure defined on a complete $\sigma$-field $\mathscr{T}$ of subsets of a point set $T$ such that $\mu(T)$ is finite.

Let $E$ denote a Banach space over the real numbers $R$, and let $E^{*}$ be its topological dual. The norms in $E$ and $E^{*}$ will be denoted by $\|\cdot\|$. For any set $A$ in $E$ or $E^{*}, \overline{\operatorname{co}} A, \operatorname{co} A, \operatorname{cl} A, \operatorname{ext} A$ will respectively stand for the closed convex hull, convex hull, norm closure and the set of extreme points of the set $A$. For any set $A$ in $E^{*}, w^{*} \operatorname{co} A, w^{*} \operatorname{cl} A$ will respectively stand for the weak* closed convex hull and weak* closure of the set $A$.

Our next set of definitions involves Bochner integrable functions; see [5, Chapter II] for details. Let $L(\mu, E)$ denote the space of all (equivalence classes of) strongly measurable functions from $T$ to $E$. Let $L_{1}(\mu, E)$ denote the space of all (equivalence classes of) $E$-valued Bochner integrable functions $f$ defined on $T$ with $\|f\|=$ $\int_{T}\|f(t)\| d \mu(t)$. Finally, let $L_{\infty}(\mu, E)$ denote the space of all (equivalence classes of) $E$-valued Bochner integrable functions defined on $T$ that are essentially bounded, i.e. such that

$$
\|f\|_{\infty}=\operatorname{ess} \sup \{\|f(t)\|: t \in T\}<\infty
$$

We shall abbreviate $L_{1}(\mu, R)$ and $L_{\infty}(\mu, R)$ to $L_{1}(\mu)$ and $L_{\infty}(\mu)$, respectively.

We shall also be concerned with functions $f: T \rightarrow E^{*}$ such that $\|f\| \in L_{\infty}(\mu)$ and $\langle x, f\rangle$ is $\mu$-measurable for every $x \in E$. Accordingly, we shall denote (the equivalence classes of) such functions as $L_{\infty}^{\mathrm{w}}\left(\mu, E^{*}\right)$. It is well known that the topological dual of $L_{1}(\mu, E)$ is $L_{\infty}^{\mathrm{w}}\left(\mu, E^{*}\right)$; see [6] for example.

We now turn to correspondence or set-valued mappings. Let $\mathscr{P}(E)$ denote the space of subsets of $E$. For any mapping $X: T \rightarrow \mathscr{P}(E)$, let $L_{1}(\mu, X(\cdot))$ denote the set $\left\{f \in L_{1}(\mu, E): f(t) \in X(T)\right.$ for almost all $t$ in $\left.T\right\}$. Similarly, for $L(\mu, X(\cdot))$, $L_{\infty}(\mu, X(\cdot))$ and $L_{\infty}^{\mathrm{w}}(\mu, X(\cdot))$.

We are now in a position to state some preliminaries on the integration of correspondences. We shall need the following definitions. The term "Gel'fand integral" follows [5, p. 53].

Definition 2.1. A set-valued mapping $P: T \rightarrow \mathscr{P}(X)$ is said to be measurable if the graph of $P,\{(t, x) \in T \times E: x \in P(t)\}$, is an element of $\mathscr{T} \otimes \mathscr{B}(E)$, where $\mathscr{B}(X)$ is the set of norm Borel subsets of $E$. A set-valued mapping $P: T \rightarrow \mathscr{P}\left(X^{*}\right)$ is said to be weak* measurable if the graph of $P$ is an element of $\mathscr{T} \otimes \mathscr{B}^{\mathrm{w}^{*}}(E)$, where $B^{w^{*}}(E)$ are the Borel subsets of $E^{*}$ in the weak* topology on $E^{*} . B^{\mathrm{w}}(E)$ shall denote the Borel subsets of $E$ in the weak topology on $E$. 
Definition 2.2. A set-valued mapping $P$ from $T$ to $E$ or $E^{*}$ is said to be integrably bounded if there exists $g \in L_{1}(\mu)$ such that $\sup \{\|x\|: x \in P(t)\} \leqslant g(t)$ for almost all $t$ in $T$. If $P(t) \subseteq K, K$ any bounded set, for all $t$ in $T, P$ is said to be strongly bounded.

Definition 2.3. The Bochner integral of a set-valued mapping $P: T \rightarrow \mathscr{P}\left(E^{*}\right)$ is defined for any $A \in \mathscr{T}$ by

$$
\int_{A} P(t) d \mu(t) \equiv\left\{\int_{A} f(t) d \mu(t): f \in L_{1}(\mu, P(\cdot))\right\}
$$

Definition 2.4. The Gel'fand integral of a set-valued mapping $P: T \rightarrow \mathscr{P}\left(E^{*}\right)$ is defined for any $A \in \mathscr{T}$ by

$$
\oint_{A} P(t) d \mu(t)=\left\{\oint_{A} f(t) d \mu(t): f \in L_{\infty}^{\mathrm{w}}(\mu, P(\cdot))\right\}
$$

where $\oint_{A} f(t) d \mu(t)$ is an element of $E^{*}$ such that

$$
\left\langle\oint_{A} f(t) d \mu(t), x\right\rangle=\int_{A}\langle f(t), x\rangle d \mu(t) \text { for all } x \text { in } E .
$$

It is clear that an integrably bounded measurable set-valued map has a nonempty Bochner integral; see Remark 1 in [15]. It can also be shown that a strongly bounded weak* measurable set-valued map has a nonempty Gel'fand integral if $E$ is separable; see Remark 2 in [15].

3. The model and results. A game consists of a set of players $T$, each of whom has a strategy set $X(t)$ and a preference relation $\succsim_{(t, x)}$ defined over $X(t) \times X(t)$. The preferences of each player depend on the actions taken by all the other players and are accordingly indexed by a play $x: T \rightarrow X(t)$ which associates with each player $t$ his action $x(t)$. We can thus summarize a game $G$ by $\left\{X(t), \succsim_{(t, x)}\right\}_{t \in T}$. A nonatomic game $G$ is one where the set of players is represented by a nonatomic measure space. Such a specification demands measurability assumptions. We thus have

Definition 3.1. A nonatomic game $G$ is a quadruple $[(T, \mathscr{T}, \mu), E, X, P]$, where

(1) $(T, \mathscr{T}, \mu)$ is a finite, complete, nonatomic measure space;

(2) $E$ is a Banach space;

(3) $X: T \rightarrow \mathscr{P}(E)$ is a measurable map; and

(4) $P: T \times L(\mu, X(\cdot)) \rightarrow \mathscr{P}(E \times E)$ is a map such that for all $x$ in $L(\mu, X(\cdot))$, the map $P(\cdot, x): T \rightarrow \mathscr{P}(E \times E)$ is measurable.

For all $t$ in $T$ and for all $x$ in $L(\mu, X(\cdot))$, we shall also use the notation $z_{1} \succsim_{(t, x)} z_{2}$ to mean $\left(z_{1}, z_{2}\right) \in P(t, x)$.

An equilibrium strategy is a play in which no player finds it worthwhile to choose another action in his strategy set given the actions of all other players. This basic 
idea, originally due to Cournot, can be formalized as

Definition 3.2. An equilibrium of a nonatomic game $G$ is given by $x^{*} \in$ $L(\mu, X(\cdot))$ such that for almost all $t$ in $T$,

$$
x^{*}(t) \succsim_{\left(t, x^{*}\right)} y \text { for all } y \text { in } X(t) .
$$

It is clear that under the generality of Definition 3.1 , there will not exist, in general, any equilibrium strategies. We shall need to assume that the strategy sets and preference relations are convex. We shall also need topological assumptions such as compactness of the strategy sets and closedness of preference relations as well as their continuous dependence on the plays. The set of admissible plays also embodies further restrictions. The existence results reported below differ essentially in the topological assumptions underlying them.

TheOREM 3.1. Let $G=[(T, \mathscr{T}, \mu), E, X, P]$ be a nonatomic game such that

(1) E is separable;

(2) for all $t$ in $T, X(t)$ is a nonempty, closed convex subset of a weakly compact set $K \subseteq E$;

(3) $P$ is defined on $T \times L_{1}(\mu, X(\cdot))$ and is such that

(i) for all $t$ in $T$ and for all $x$ in $L_{1}(\mu, X(\cdot)), \succsim_{(t, x)}$ is a reflexive, transitive and convex relation;

(ii) for all $t$ in $T$, the graph of $P(t, \cdot)$ given by

$$
\left\{\left(x, z_{1}, z_{2}\right) \in L_{1}(\mu, X(\cdot)) \times X(t) \times X(t): z_{1} \succsim_{(t, x)} z_{2}\right\}
$$

is closed in the product topology, where $L_{1}(\mu, X(\cdot))$ and $X(t)$ are each endowed with their relative weak topologies.

Then $G$ has an equilibrium.

The first point to be noticed about Theorem 3.1 is that, despite condition (1), it is valid for the spaces $L_{\infty}(\mu)$ and $\operatorname{rca}(S)$, the set of all regular countably additive scalar-valued set functions defined on the Borel $\sigma$-field on a compact Hausdorff space $S$ with a countable base. The reason is essentially contained in the requirement that, for all $t$ in $T, X(t)$ is contained in a weakly compact subset of $E$; see condition (2) of Theorem 3.1. We can thus state

COROllaRy 3.1. Theorem 3.1 is valid for the nonseparable spaces $L_{\infty}(\mu)$ and $\operatorname{rca}(S)$.

It is clear that condition (2) of Theorem 3.1 imposes a strong uniformity requirement on the distribution of strategy sets. A natural question arises as to the extent to which it can be weakened. In the author's earlier work [14], the existence of equilibrium strategies was shown under the requirement that the measurable map $X$ is integrably bounded and that, for all $t$ in $T, X(t)$ is nonempty, convex and weakly compact. However, in order to cope with such a general specification on the strategy sets, we needed the additional assumption that the dual of $E$ possess the RadonNikodým property (RNP). Our next result shows the extent to which we can generalize condition (2) without assuming RNP. 
THEOREM 3.2. Theorem 3.1 is valid with (2a) substituted for (2) where

(2a) $X$ is an integrably bounded map with weakly compact values and such that for all $\varepsilon>0$, there exist $T_{\varepsilon} \in \mathscr{T}, \mu\left(T-T_{\varepsilon}\right)<\varepsilon$, a bounded uniformly integrable subset $J_{\varepsilon}$ of $L_{1}\left(\left.\mu\right|_{T_{s}}\right)$, and a weakly compact subset $K_{\varepsilon} \subseteq E$ such that $x \in L_{1}(\mu, P(\cdot))$ implies $x(t)=\sum_{n} \lambda_{n} f_{n}(t) x_{n}$ for almost all $t$ in $T$, with scalars $\lambda_{n}$ such that $\sum_{n}\left|\lambda_{n}\right| \leqslant 1$, $f_{n} \in J_{\varepsilon}$ and $x_{n} \in K_{\varepsilon}$.

The generality of $(2 a)$ can best be appreciated in steps. The first extension of the assumption that all the strategy sets sit in the same weakly compact subset $K$ of $E$ is to allow this compact set to change in a manner which is regulated by an integrable function $f$; i.e. $X(t) \subseteq f(t) K$. Such an assumption occurs, for example, in Castaing's work; see [3, Corollary V.4]. The next step is to allow this change to be regulated not by one integrable function but by a countably infinite number chosen from a bounded, integrable family $J$; i.e. $X(t) \subseteq \sum_{n} \lambda_{n} f_{n}(t) x_{n}$, where $\lambda_{n}$ are scalars such that $\sum_{n}\left|\lambda_{n}\right| \leqslant 1, f_{n} \in J$ and $x_{n} \in K$. The final step is to allow for the fact that the above representation does not obtain for the strategy set of every trader and that there is a subset of traders $T_{\varepsilon}$ with $\mu\left(T_{\varepsilon}\right)<\varepsilon$ whose strategy sets do not fit in this mold. Once we allow $\varepsilon$ to take on arbitrarily small values and index $J$ and $K$ by $\varepsilon$, we obtain condition $(2 a)$.

It is by no means clear that Theorem 3.2 extends to the spaces $L_{\infty}(\mu)$ and $\operatorname{rca}(S)$. Thus it is worthwhile to have a result which pertains primarily to such spaces. It is of interest, though maybe not surprising, that we can present such a result without insisting on weak compactness of the strategy sets but only that they be norm bounded and weak* closed.

THEOREM 3.3. Let $G=\left[(T, \mathscr{T}, \mu), E^{*}, X, P\right]$ be a nonatomic game such that

(1) $E^{*}$ is the dual of a separable Banach space, and $\mu$ is such that $L_{1}(\mu)$ is separable;

(2) the measurability assumptions on $X$ and $P$ in Definition 3.1 are the interpreted with respect to the weak* Borel sets on $E^{*}$;

(3) for all $t$ in $T, X(t)$ is a nonempty, convex, weak* closed subset of a norm bounded set $K \subset E^{*}$;

(4) $P$ is defined on $T \times L_{\infty}^{\mathrm{w}}(\mu, X(\cdot))$ and is such that

(i) for all $t$ in $T$ and for all $x$ in $L_{\infty}^{\mathrm{w}}(\mu, X(\cdot)), \succsim_{(t, x)}$ is a reflexive, transitive and convex relation;

(ii) for all $t$ in $T$, the graph of $P(t, \cdot)$ given by

$$
\left\{x, z_{1}, z_{2} \in L_{\infty}^{\mathrm{w}}(\mu, X(\cdot)) \times X(t) \times X(t): z_{1} \succsim_{(t, x)} z_{2}\right\}
$$

is closed in the product topology, where $L_{\infty}^{\mathrm{w}}(\mu, X(\cdot))$ is endowed with the relative weak ${ }^{*}$ topology, i.e. $\sigma\left(L_{\infty}^{\mathrm{w}}\left(\mu, E^{*}\right), L_{1}(\mu, E)\right)$ and $X(t)$ with relative Mackey topology, i..e $\tau\left(E^{*}, E\right)$.

Then $G$ has an equilibrium.

The reader should note that Theorems 3.1-3.3 do not depend on the nonatomicity of the measure $\mu$. 
We now turn to the existence of pure strategy equilibria, i.e. Cournot-Nash equilibria in which almost every player chooses an extreme point in his strategy set. Here, following Schmeidler [19], we shall assume that the preferences of each player are represented by a linear payoff function which satisfies the aggregation axiom in the sense of [7]; i.e. it depends on the average response of all the other players. It is at this point that integration becomes an essential aspect of the theory. Our first result is

THEOREM 3.4. Let $G=[(T, \mathscr{T}, \mu), E, X, u]$ be a nonatomic game such that $E$ and $X$ satisfy conditions (1) and (2) of Theorem 3.1, and $u: T \times X(t) \times E \rightarrow R_{+}$is a map such that

(i) for all $x \in E, u(\cdot, \cdot, x)$ is a Borel measurable function on $\{(t, x) \in T \times X(t)$ : $x \in X(t)\}$;

(ii) for all $t$ in $T, x \in E, u(t, \cdot, x)$ is linear and weakly continuous on $X(t)$;

(iii) for all $t$ in $T$, for all $x \in X(t), u(t, x, \cdot)$ is weakly continuous on $E$.

Then $G$ has an approximate, pure strategy equilibrium; i.e. $\forall \varepsilon>0$, there exists $x^{*} \in L_{1}(\mu$, ext $X(\cdot))$ such that for almost all $t$ in $T,\left\|u\left(t, x^{*}(t), x_{T}^{*}\right)-u^{*}(t)\right\|<\varepsilon$, where $x_{T}^{*}=\int_{T} x^{*}(t) d \mu(t)$ and $u_{t}^{*}=\operatorname{Max}_{z \in X(t)} u\left(t, z, x_{T}^{*}\right)$.

As will become clear in the proofs below, Theorem 3.4 relies in an essential way on the validity of Theorem 3.1. Accordingly, we can also show

COROllary 3.2. Theorem 3.4 is valid for the nonseparable spaces $L_{\infty}(\mu)$ and $\operatorname{rca}(S)$ or, alternatively, with condition (2a) of Theorem 3.2 substituted for condition (2) of Theorem 3.1.

All that remains is for us to obtain an analogue of Theorem 3.4 for the setup of Theorem 3.3, where the game is defined on the dual $E^{*}$ of a separable Banach space. The principal difficulty here is our formalization of a play as an element of $L_{\infty}^{\mathrm{w}}(\mu, X(\cdot))$. As such, a play is weakly measurable and is not necessarily Bochner integrable. However, we can appeal to results on the Gel'fand integral of a set-valued mapping; see [15]. We can thus state our final result:

TheOREM 3.5. Let $G=\left[(T, \mathscr{T}, \mu), E^{*}, X, u\right]$ be a nonatomic game such that $E^{*}$ and $X$ satisfy conditions (1)-(3) of Theorem 3.3 and $u: T \times X(t) \times E^{*} \rightarrow R_{+}$is a map such that

(i) for all $x \in E^{*}, u(t, \cdot, \cdot, x)$ is a weak ${ }^{*}$ Borel measurable function on $\{(t, x) \in$ $T \times X(t): x \in X(t)\}$;

(ii) for all $t$ in $T, u(t, \cdot, x)$ is linear and $\tau\left(E^{*}, E\right)$-continuous on $X(t)$;

(iii) for all $t$ in $T$, for all $x \in X(t), u(t, x, \cdot)$ is weak* continuous on $E^{*}$.

Then $G$ has an approximate pure strategy equilibrium, i.e. $\forall \varepsilon>0$, there exists $x^{*} \in L_{\infty}^{\mathrm{w}}(\mu$, ext $X(\cdot))$ such that, for almost all $t$ in $T$,

$$
\left\|u\left(t, x^{*}(t), x_{T}^{*}\right)-u^{*}(t)\right\|<\varepsilon,
$$

where $x_{T}^{*}=\oint_{T} x^{*}(t) d \mu(t)$ and $u_{t}^{*}=\operatorname{Max}_{z \in X(t)} u\left(t, z, x_{T}^{*}\right)$. 
We conclude this section with three open problems. First, are Theorems 3.4 and 3.5 vaild without the requirement that the preferences be represented as payoff functions? It is clear that the nature of approximate pure strategy equilibria has to be modified. A principal difficulty seems to be the continuity of the best response sets, i.e. $B\left(t, x_{T}\right)$; see [12, paragraph 5, p. 30]. Second, are all of our theorems valid when the preferences are nonordered? Third, can the assumption on $\mu$ in Theorem 3.3 be relaxed?

4. Proofs. We begin with the proof of Theorem 3.1. It is an application of the Fan-Glicksberg $[9,10]$, fixed-point theorem to a map $\alpha$ which takes $L_{1}(\mu, X(\cdot))$ to subsets of $L_{1}(\mu, X(\cdot))$. We endow $L_{1}(\mu, X(\cdot))$ with its relative weak topology. We use Diestel's theorem [4] to show that the domain of this map is compact.

Proof of Theorem 3.1. Consider the map $\alpha: L_{1}(\mu, X(\cdot)) \rightarrow \mathscr{P}\left(L_{1}(\mu, X(\cdot))\right)$ where

$$
\begin{aligned}
& \alpha(x)=\left\{y \in L_{1}(\mu, E): y(t) \in B(t, x) \text { for almost all } t \text { in } T\right\} \\
& B(t, x)=\left\{y(t) \in X(t): y(t) \succsim_{(t, x)} z \text { for all } z \in X(t)\right\} .
\end{aligned}
$$

It is clear that any fixed point of the map $\alpha(\cdot)$ yields an equilibrium of our nonatomic game. Thus all we have to verify is that $\alpha(\cdot)$ satisfies the conditions required by the Fan-Glicksberg theorem. We do this in a series of claims.

Claim 1. $L_{1}(\mu, X(\cdot))$ is weakly compact.

Since $X(t) \subseteq K$ for all $t$ in $T, K$ weakly compact and hence bounded [8, II.3.20], we can assert that the set $L_{1}(\mu, X(\cdot))$ is bounded and uniformly integrable. We can thus apply Theorem 2 in [4] to establish our claim.

Claim 2. $L_{1}(\mu, X(\cdot))$ is nonempty and convex.

We have already observed that $L_{1}(\mu, X(\cdot))$ is convex. Since the graph of $X(\cdot)$ belongs to $\mathscr{T} \otimes \mathscr{B}(E)$, Theorem III.22 in [3] guarantees us a measurable selection. Since $X(t)$ is uniformly bounded, such a selection is certainly an element of $L_{1}(\mu, X(\cdot))$.

Claim 3. For each $x$ in $L_{1}(\mu, X(\cdot))$ and for almost all $t$ in $T, B(t, x) \neq \varnothing$.

Since $X(t)$ is closed and a subset of a weakly compact set $K$, it is also weakly compact [8, I.V.7a]. For all $x$ in $L_{1}(\mu, X(\cdot)), \succsim_{(t, x)}$ is reflexive, transitive and weakly closed. We can thus apply the arguments of [12, Theorem 3, p. 29] to assert that $B(t, x) \neq \varnothing$.

Claim 4. For each $x$ in $L_{1}(\mu, X(\cdot))$, the graph of $B(\cdot, x)$ belongs to $\mathscr{T} \otimes \mathscr{B}(E)$.

The proof of this claim is based on an argument in Hildenbrand [11, p. 615].

Since the graph of the correspondence $X(\cdot)$ belongs to $\mathscr{T} \otimes \mathscr{B}(E)$, we can appeal to Definition III.21 and Theorem III.22 in [3] to assert the existence of a sequence $\left(f_{n}(\cdot)\right)$ of measurable selections of $X(\cdot)$ such that for every $t$ in $T, f_{n}(t)$ is norm dense in $A(t)$.

Now let $B_{n}(t, x)=\left\{y(t) \in X(t): y(t) \succsim_{(t, x)} f_{n}(t)\right\}$. Certainly the graph of $\Gamma(\cdot)$ $\equiv\left(X(\cdot), f_{n}(\cdot)\right)$ belongs to $\mathscr{T} \otimes \mathscr{B}(E \times E)$. Since the graph of $P(\cdot, x)$ also belongs to $\mathscr{T} \otimes \mathscr{B}(E \times E)$, we can assert that the graph of $P(\cdot, x) \cap \Gamma(\cdot)$ and hence of $B_{n}(\cdot, x)$ belongs to $\mathscr{T} \otimes \mathscr{B}(E \times E)$. 
Now we show that $B(t, x)=\bigcap_{n} B_{n}(t, x)$ for almost all $t$ in $T$. Certainly $B(t, x) \subset$ $B_{n}(t, x)$ for every $n$. Suppose $z \in \bigcap_{n} B_{n}(t, x)$, but $z \notin B(t, x)$. Then there exists $y \in X(t)$ such that $y \succ_{(t, x)} z$. Since the norm topology is stronger than the weak topology [8, V.3.5], we can find some $f_{n}^{k}(t)$ arbitrarily weakly close to $y$. But $\succsim_{(t, x)}$ is weakly closed, and hence $\succ_{(t, x)}$ is weakly open; and we obtain a contradiction.

We thus obtain

$$
\{(t, y) \in T \times E: y \in B(t, x)\}=\bigcap_{n}\left\{(t, y) \in T \times E: y \in B_{n}(t, x)\right\},
$$

and the proof of our claim is complete.

Claim 5. For each $x$ in $L_{1}(\mu, X(\cdot)), \alpha(x)$ is nonempty and convex.

Convexity follows routinely from the properties of $\succsim_{(t, x)}$. Nonemptiness follows from an application of Theorem III.22 in [3].

Claim 6. For almost all $t$ in $T, B(t, \cdot)$ has a closed graph in $L_{1}(\mu, E) \times E$.

Let a net $\left(y^{\nu}, x^{\nu}\right)$ converge in the weak product topology to $(y, x)$ where $y^{\nu} \in B\left(t, x^{\nu}\right)$. We have to show that $y \in B(t, x)$. Suppose not; i.e. there exists $z \in X(t)$ such that $z \succ_{(t, x)} y$. Since $\succ_{(t, x)}$ is weakly open, there exists a large enough $\nu$ such that $z \succ_{(t, x)} y^{\nu}$, and we obtain a contradiction.

Claim 7. The graph of $\alpha(\cdot)$ is weakly closed in $L_{1}(\mu, E) \times L_{1}(\mu, E)$.

Since weakly sequentially closed subsets of a weakly compact set are weakly compact (by the Eberlein-Smulian theorem) and hence weakly closed, we need only consider a sequence $\left(z^{n}, x^{n}\right)$ which tends weakly to $(z, x)$. Now suppose that $y \notin \alpha(x)$; i.e. there exists $S \in \mathscr{T}, \mu(S)>0$ such that $y(t) \notin B(t, x)$ for all $t$ in $S$. Let

$$
\alpha_{S}(x)=\left\{z \in L_{1}\left(\left.\mu\right|_{S},\left.X\right|_{S}\right): z(t) \in B(t, x) \text { for almost all } t \in S\right\} .
$$

Just as in Claim 5, $\alpha_{S}(x)$ is nonempty and convex. It is also closed. This can be seen by considering a sequence $z_{\nu} \in \alpha_{S}(x)$ which converges to $z$; i.e. $\lim _{\nu \rightarrow \infty}\left\|z_{\nu}-z\right\|=0$. This implies that for almost all $t$ in $T, \lim _{\nu \rightarrow \infty}\left\|z_{\nu}(t)-z(t)\right\|=0$. Our assertion now follows from the closedness of $B(t, x)$ established in Claim 6. Let $y_{S}$ be the restriction of $y$ to $\left.\mu\right|_{s}$. By hypothesis $y_{S} \notin \alpha_{S}(x)$. We can now apply the HahnBanach theorem [8, V.2.10] to assert that there exists a nonzero, continuous linear functional $f \in\left(L_{1}(\mu, E)\right)^{*}$ such that $f\left(y_{S}\right)>f(z)$ for all $z \in \alpha_{S}(x)$. But $\left(L_{1}(\mu, E)\right)^{*}=L_{\infty}^{\mathrm{w}}\left(\mu, E^{*}\right)$; see [6] for example. We may thus write

$$
\int_{S}\langle y(t), f(t)\rangle d \mu(t)>\int_{S}\langle z(t), f(t)\rangle d \mu(t) \quad \forall z \in \alpha_{S}(x)
$$

Since $y_{n}$ converges weakly to $y$, certainly

$$
\lim _{n \rightarrow \infty} \int_{S}\left\langle y^{n}(t), f(t)\right\rangle d \mu(t)=\int_{S}\langle y(t), f(t)\rangle d \mu(t) .
$$

Since $y^{n}(t) \in K, K$ weakly compact, for all $t$ in $T$ and for all $n$, and $\|f\| \in L_{\infty}(\mu)$, $\left\langle y^{n}(t), f(t)\right\rangle$ is integrably bounded. We can thus apply Proposition 4.1 in [1] to conclude that

$$
\lim _{n \rightarrow \infty} \int_{S}\left\langle y^{n}(t), f(t)\right\rangle d \mu(t) \in \int_{S} \operatorname{Limsup}_{n \rightarrow \infty}\left\langle y^{n}(t), f(t)\right\rangle d \mu(t) .
$$


Since $f(t) \in E^{*}$, certainly $\operatorname{Limsup}_{n \rightarrow \infty}\left\langle y^{n}(t), f(t)\right\rangle=\left\langle\operatorname{Limsup}_{n \rightarrow \infty} y^{n}(t), f(t)\right\rangle$. But, by Claim 6, each limit point of $y^{n}(t) \in B(t, x)$ for almost all $t$ in $T$. We thus have a contradiction to (1), and the proof of the claim is complete.

We can now apply the Fan-Glicksberg theorem to complete the proof of Theorem 3.1. Q.E.D.

Proof of Corollary 3.1. Consider the space $L_{\infty}(\mu)$. By Rosenthal's theorem [5, Theorem 13, p. 252], every weakly compact set is norm separable. Thus the strategy set correspondence $X(\cdot)$ maps into a separable subset of $L_{\infty}(\mu)$. Now apply all the arguments in the proof of Theorem 3.1.

The fact that weakly compact subsets of $\operatorname{rca}(S)$ are norm separable follows from Exercise 9.5.113 in Wilansky [22, p. 145]. Q.E.D.

Proof of Theorem 3.2. Note that the only place where we needed the fact that for all $t$ in $T, X(t) \subseteq K, K$ weakly compact, was in the proof of Claim 1 in the proof of Theorem 3.1. This condition was used in applying Diestel's theorem. However, under the alternative condition (2a), we can appeal to Corollary 5 in Diestel [4] to establish Claim 1. The remaining argument in the proof of Theorem 3.1 applies unchanged. Q.E.D.

REMARK. It should be clear to the reader why the argument in the proof of Corollary 3.1 fails to extend to the establishment of Theorem 3.2 for the spaces $L_{\infty}(\mu)$ and $\operatorname{rca}(S)$.

Next, we turn to the proof of Theorem 3.3. Its basic outline is the same as the proof of Theorem 3.1, other than the fact that we now work with the weak* topology on $L_{\infty}^{\mathrm{w}}(\mu, X(\cdot))$. As such, we can no longer use Diestel's results. We have to fall back instead on results in Castaing and Valadier [3].

Proof of TheOrem 3.3. Consider the map $\alpha: L_{\infty}^{\mathrm{w}}(\mu, X(\cdot)) \rightarrow \mathscr{P}\left(L_{\infty}^{\mathrm{w}}(\mu, X(\cdot))\right)$,

$$
\alpha(x)=\left\{y \in L_{\infty}^{\mathrm{w}}(\mu, X): y(t) \in B(t, x) \text { for almost all } t \text { in } T\right\},
$$

and $B(t, x)$ as in the proof of Theorem 3.1. We shall show that $\alpha(\cdot)$ has a fixed point.

Claim $1 . L_{\infty}^{\mathrm{w}}(\mu, X)$ is weak* compact.

Apply Theorem V.1 in [3].

Claim 2. $L_{\infty}^{\mathrm{w}}(\mu, X)$ is nonempty and convex.

Convexity is straightforward. Nonemptiness follows from Theorem III.37 in [3]. Note, however, that the theorem requires separability which we have not assumed for our underlying Banach space $E^{*}$. However, for all $t$ in $T, X(t) \subseteq K, K$ is weak* closed, and hence, without any loss of generality, can be chosen to be weak* compact. We can now appeal to Theorem 9.3 in Wilansky [22, p. 143], to assert that $K$ is separable and, hence, a Souslin subset of $E$.

Claim 3. For each $x$ in $L_{\infty}^{\mathrm{w}}(\mu, X(\cdot))$, and for almost all $t$ in $T, B(t, x) \neq \varnothing$ and is weak* closed.

Since the set $\left\{\left(z_{1}, z_{2}\right) \in X(t) \times X(t): z_{1} \succsim_{(t, x)} z_{2}\right\}$ is convex and Mackey closed, it is also weak* closed; see [22, 8.3.6, p. 111]. Thus $\succ_{(t, x)}$ is weak* open, and the proof can be completed as in [12, Theorem 3, p. 29]. 
Claim 4. For each $x$ in $L_{\infty}^{\mathrm{w}}(\mu, X(\cdot))$, the graph of $B(\cdot, x)$ belongs to $\mathscr{T} \otimes \mathscr{B}^{\mathrm{w}^{*}}\left(E^{*}\right)$.

The proof follows that of Claim 5 in the proof of Theorem 3.1. The principal difference to be noted is that we are now working with the weak* Borel sets on $E^{*}$ rather than the norm Borel sets. We have already observed earlier that $\succ_{(t, x)}$ is weak* open. Thus the proof of Claim 5 in the proof of Theorem 3.1 applies.

Claim 5. For each $x$ in $L_{\infty}^{\mathrm{w}}(\mu, X(\cdot)), \alpha(x)$ is nonempty and convex.

This can be established as in the proof of Claim 6 in the proof of Theorem 3.1. The fact that we can legitimately appeal to Theorem III.22 in [3] follows from the fact that $K$ is Souslin, as in the proof of Claim 2 above.

Claim 6. For almost all $t$ in $T, B(t, \cdot)$ has a closed graph in $L_{\infty}^{\mathrm{w}}\left(\mu, E^{*}\right) \times E^{*}$.

Once we recall that $\succ_{(t, x)}$ is weak* open, the argument is identical to that of the proof of Claim 7 in the proof of Theorem 3.1.

Claim 7. For each $x$ in $L_{\infty}^{\mathrm{w}}(\mu,, X(\cdot)), \alpha(x)$ is closed in the weak* topology on $L_{\infty}^{\mathrm{w}}\left(\mu, E^{*}\right)$, i.e. $\sigma\left(L_{\infty}^{\mathrm{w}}\left(\mu, E^{*}\right), L_{1}(\mu, E)\right)$.

From Claim 3, we know that the map $B(\cdot, x)$ has nonempty and weak* compact values. They are also convex. We can thus appeal to Theorem III.37 in [3] and to Claim 4 above to assert that $B(\cdot, x)$ is scalarly measurable. We can now appeal to Theorem V.1 in [3] to assert that $\alpha(x)$ is weak* closed.

Claim 8. The graph of $\alpha(\cdot)$ is closed in the product topology on $L_{\infty}^{\mathrm{w}}\left(\mu, E^{*}\right) \times$ $L_{\infty}^{\mathrm{w}}\left(\mu, E^{*}\right)$.

Observe first that under condition (1) of Theorem 3.3, $L_{1}(\mu, E)$ is separable. We can now appeal to [22, Theorem 9.5.3] to assert that the graph of $\alpha(\cdot)$ is a subset of a compact metric space. We can thus consider a sequence $\left(x^{n}, y^{n}\right)$ which converges in the weak* topology to $(x, y)$. Suppose that $y \notin \alpha(x)$; i.e. there exists $S \in \mathscr{T}$, $\mu(S)>0$ such that $y(t) \notin B(t, x)$ for all $t$ in $S$. Let $\alpha_{S}(x)=\left\{z \in L_{\infty}^{\mathrm{w}}\left(\left.\mu\right|_{S},\left.X\right|_{S}\right)\right.$ : $z(t) \in B(t, x)$ for almost all $t$ in $S\}$. From Claims 5 and 7 above, $\alpha_{S}(x)$ is nonempty, convex and weak* closed. Let $y_{S}$ be the restriction of $y$ to $\left.\mu\right|_{s}$. By hypothesis $y_{S} \notin \alpha_{S}(x)$. We can now apply the Hahn-Banach theorem [8, V.2.10] to assert that there exists a nonzero, continuous linear functional $f \in L_{1}(\mu, E), f \neq 0$, such that $f\left(y_{S}\right)>f(z)$ for all $z \in \alpha_{S}(x)$; i.e.

$$
\int_{S}\langle y(t), f(t)\rangle d \mu(t)>\int_{S}\langle z(t), f(t)\rangle d \mu(t) \quad \forall z \in \alpha_{S}(x) .
$$

Since $y^{n}$ converges in the weak* topology to $y$, certainly

$$
\operatorname{Lim}_{n \rightarrow \infty} \int_{S}\left\langle y^{n}(t), f(t)\right\rangle d \mu(t)=\int_{S}\langle y(t), f(t)\rangle d \mu(t)
$$

Since $y^{n}$ is integrably bounded and $f(\cdot)$ is Bochner integrable, certainly there exists $g \in L_{1}\left(\left.\mu\right|_{S}\right)$ such that $\left\langle y^{n}(t), f(t)\right\rangle \mid \leqslant g(t)$ for almost all $t$ in $S$. We can now apply Proposition 4.1 in [1] to conclude that

$$
\operatorname{Lim}_{n \rightarrow \infty} \int_{S}\left\langle y^{n}(t), f(t)\right\rangle d \mu(t) \in \int_{S} \operatorname{Limsup}_{n \rightarrow \infty}\left\langle y^{n}(t), f(t)\right\rangle d \mu(t)
$$


Since $\langle\cdot, f(t)\rangle$ is weak* continuous, certainly

$$
\limsup _{n \rightarrow \infty}\left\langle y^{n}(t), f(t)\right\rangle=\left\langle\underset{n \rightarrow \infty}{\operatorname{Limsup}} y^{n}(t), f(t)\right\rangle .
$$

But by Claim 6, every limit point of $y(t)$ is an element of $B(t, x)$ for almost all $t$ in $T$, and we have a contradiction to (1) and the proof of Claim 8 is complete.

We can now apply the Fan-Glicksberg theorem to complete the proof of Theorem 3.3. Q.E.D.

We now turn to the proofs of our results on the existence of approximate pure-strategy equilibria. The proofs are modelled on the proof of Theorem 2 in [19]. However, there are two basic differences. First, we have to appeal to results in [15] on the integration of set-valued mappings whose range does not have RNP. Second, we no longer assume that ext $X(\cdot)$ is measurable but, thanks to Leese [20, p. 879], deduce it as a consequence of our other assumptions.

Proof OF THEOREM 3.4. Observe, first of all, that the hypotheses of Theorem 3.4 imply those of Theorem 3.1. The only part to be verified relates to preferences. However, all we have to do in this connection is to consider a mapping $P$ : $T \times L_{\infty}(\mu, X(\cdot)) \rightarrow \mathscr{P}(E \times E)$ such that for all $t$ in $T$, for all $x$ in $L_{\infty}(\mu, X(\cdot))$,

$$
u\left(t, z_{1}, x\right) \geqslant u\left(t, z_{2}, x\right) \Leftrightarrow\left(z_{1}, z_{2}\right) \in P(t, x) \Leftrightarrow z_{1} \succsim_{(t, x)} z_{2} .
$$

We can now appeal to Theorem 3.1 to assert the existence of $x \in L_{\infty}(\mu, X(\cdot))$ such that for almost all $t$ in $T, x(t) \in B\left(t, x_{T}\right)$. Let

$$
B^{e}\left(t, x_{T}\right)=\left\{x \in \operatorname{ext} X(t): u\left(t, x, x_{T}\right) \geqslant u\left(t, y, x_{T}\right) \forall y \in \operatorname{ext} X(t)\right\} .
$$

We can assert

Claim 1. For all $t$ in $T, B\left(t, x_{T}\right)=\overline{\operatorname{co}} B^{e}\left(t, x_{T}\right)$.

Since $X(t)$ is a nonempty, weakly compact and convex subset of $E$, we can appeal to a corollary of the Krein-Milman theorem [16, Corollary 11.2.3] to assert that there exists $z \in$ ext $X(t)$ such that

$$
u\left(t, z, x_{T}\right)=m_{t}^{*}=\operatorname{Max}_{q \in X(t)} u\left(t, q, x_{T}\right) .
$$

Since $z \in B^{e}\left(t, x_{T}\right)$, it is clear that $B^{e}\left(t, x_{T}\right) \subseteq B\left(t, x_{T}\right)$. Since $B\left(t, x_{T}\right)$ is closed and convex, $\operatorname{co} B^{e}\left(t, x_{T}\right) \subseteq B\left(t, x_{T}\right)$. Now suppose the containment is strict; i.e. there exists $z \in B\left(t, x_{T}\right)$ and $z \notin \overline{\operatorname{co}} B^{e}\left(t, x_{T}\right)$. By the Krein-Milman theorem [16, Theorem 11.2.1], there exist $z_{1}$ and $z_{2}$ in ext $X(t)$ and $0<\lambda<1$ such that $z=\lambda z_{1}+(1-\lambda) z_{2}$. If both $z_{1}$ and $z_{2}$ are in $B^{e}\left(t, x_{T}\right)$, then by linearity of $u\left(t, \cdot, x_{T}\right)$, so is $z$. Thus, suppose at least one $z_{i} \notin B^{e}\left(t, x_{T}\right)$. But then $u\left(t, z, x_{T}\right)<$ $m^{*}$, a contradiction.

Claim 2. $\int_{T} B\left(t, x_{T}\right) d \mu(t)=\operatorname{cl} \int_{T} B^{e}\left(t, x_{T}\right) d \mu(t)$.

Since we are working in a separable Banach space $E$, the weak topology on $K$ is metrizable [8, V.6.3]. In addition, the norm Borel $\sigma$-algebra is identical to the weak Borel $\sigma$-algebra [17, Theorem 2.5(b)]. We can thus appeal to Theorem 3.5 in [13] to assert that $X(\cdot)$ is measurable in the sense required by Theorem 8.3 in [20]. Since all the remaining hypotheses of this theorem also hold, we can assert that ext $X(\cdot)$ is measurable in the sense that its graph belongs to $\mathscr{T} \otimes \mathscr{B}^{\mathrm{w}}(X)$. A further appeal to 
[17, Theorem 2.5(b)] allows us to assert that ext $X(\cdot)$ is measurable in the sense required for Theorem III.39 in [3]. The other hypotheses of this theorem are also fulfilled, allowing us to conclude that $B^{e}(t, x)$ has a measurable graph in the sense required for Theorem 1 in [15]. The other hypotheses of this theorem are also trivially fulfilled. The proof of our claim is thus complete.

Now from Claim 2 we can assert that for all $\delta>0$, there exists $y \in L_{1}(\mu$, ext $X(\cdot))$ such that for almost all $t$ in $T, y(t) \in B^{e}\left(t, x_{T}\right)$ and $\left\|y_{T}-x_{T}\right\|<\delta$. This implies, by weak continuity of $u(t, x(t), \cdot)$, that for all $\varepsilon>0$ and for almost all $t$ in $T$,

$$
\left\|u\left(t, x(t), x_{T}\right)-u_{t}^{*}\right\|<\varepsilon / 2
$$

see [11, Corollary, p. 30]. But from Claim 1, for almost all $t$ in $T$,

$$
u\left(t, y(t), x_{T}\right)=u\left(t, x(t), x_{T}\right) .
$$

A further appeal to weak continuity of $u(t, x(t), \cdot)$ allows us to assert that for almost all $t$ in $T$,

$$
\left\|u\left(t, y(t), x_{T}\right)-u\left(t, y(t), y_{T}\right)\right\|<\varepsilon / 2 .
$$

The proof of the theorem is now complete. Q.E.D.

Proof of Corollary 3.2. Apply Corollary 3.1 and Theorem 3.2 in place of Theorem 3.1 in the proof of Theorem 3.4. All the arguments go through. Q.E.D.

Proof OF TheOREM 3.5. It is clear that the hypotheses of Theorem 3.5 imply those of Theorem 3.3. We can thus assert the existence of $x \in L_{\infty}^{\mathrm{w}}(\mu, X(\cdot))$ such that for almost all $t$ in $T, x(t) \in B\left(t, x_{T}\right)$. Let $B^{e}\left(t, x_{T}\right)$ be as in the proof of Theorem 3.4. We now have

Claim 1. For all $t$ in $T, B\left(t, x_{T}\right)=\mathrm{w}^{*} \operatorname{co} B^{e}\left(t, x_{T}\right)$.

This is established in a manner entirely analogous to the proof of Claim 1 in the proof of Theorem 3.4 .

Claim 2. $\oint_{T} B\left(t, x_{T}\right) d \mu(t)=\mathrm{w}^{*} \mathrm{cl} \phi_{T} B^{e}\left(t, x_{T}\right) d \mu(t)$.

As argued in the proof of Claim 2 in the proof of Theorem 3.3, we can assume that $X(\cdot)$ has a Souslin range space. We can thus appeal to Theorem 3.5 in [13] and to Theorem 8.3 in [20] to assert that the graph of ext $X(\cdot)$ belongs to $\mathscr{T} \otimes \mathscr{B}^{w^{*}}(X)$. We can now appeal to Theorem III.39 in [3] to assert that the graph of $B^{e}\left(\cdot, x_{T}\right)$ belongs to $\mathscr{T} \otimes \mathscr{B}^{\mathrm{w}^{*}}(X)$. A final appeal to Corollary 1 in [15] completes the proof of our claim.

The rest of the argument in the proof of Theorem 3.4 applies without any essential changes. Q.E.D.

ACKNowledgement. I am especially indebted to Roko Aliprantis, Don Brown, S. S. Khurana, Peter Loeb, Andreu Mas-Colell, Joe Ostroy, Salim Rashid, Rajiv Vohra and Nicholas Yannelis for many stimulating discussions, and to an anonymous referee for his comments. Errors are, of course, solely mine.

\section{REFERENCES}

1. R. J. Aumann, Integrals of set-valued functions, J. Math. Anal. Appl. 12 (1965), 1-12.

2. T. F. Bewley, The equality of the core and the set of equilibria in economies with infinitely many commodities and a continuum of agents, Internat. Econom. Rev. 14 (1974), 383-396.

3. C. Castaing and M. Valadier, Convex analysis and measurable multifunctions, Lecture Notes in Math., vol. 580, Springer-Verlag, New York, 1977. 
4. J. Diestel, Remarks on weak compactness in $L_{1}(\mu, X)$, Glasgow Math. J. 18 (1977), 87-91.

5. J. Diestel and J. J. Uhl, Jr., Vector measures, Math. Surveys, No. 15, Amer. Math. Soc., Providence, R. I., 1977.

6. N. Dincleanu, Linear operations on $L^{p}$-spaces, Vector and Operator Valued Measures and Applications (D. H. Tucker and H. B. Maynard, eds.), Academic Press, New York, 1973.

7. P. Dubey, A. Mas-Colell and M. Shubik, Efficiency properties of strategic market games: An axiomatic approach, J. Econom. Theory 22 (1980), 339-362.

8. N. Dunford and J. T. Schwartz, Linear operators: Part I, Wiley, New York, 1958.

9. K. Fan, Fixed points and minimax theorems in locally convex linear spaces, Proc. Nat. Acad. Sci. U.S.A. 38 (1952), 121-126.

10. I. L. Glicksberg, A further generalization of the Kakutani fixed point theorem with applications to Nash equilibrium points, Proc. Amer. Math. Soc. 3 (1952), 170-174.

11. W. Hildenbrand, Existence of equilibria for economies with production and a measure space of consumers, Econometrica 38 (1970), 608-623.

12. Core and equilibria of a large eocnomy, Princeton Univ. Press, Princeton, N. J., 1974.

13. C. J. Himmelberg, Measurable relations, Fund. Math. 87 (1975), 53-72.

14. M. Ali Khan, Equilibrium points of nonatomic games over a nonreflexive Banach space, J. Approx. Theory 43 (1985), 370-376.

15. __ On the integration of set-valued mappings in a nonreflexive Banach space. II, Johns Hopkins Working Paper No. 109, September 1982 (Simon Stevins, forthcoming).

16. R. Larsen, Functional analysis, Dekker, New York, 1973.

17. P. Masani, Measurability and Pettis integration in Hilhert spaces, J. Reine Angew. Math. 297 (1978), 92-135.

18. A. Mas-Colell, A model of equilibrium with differentiated commodities, J. Math. Econom. 2 (1975), 263-295.

19. D. Schmeidler, Equilibrium points of nonatomic games, J. Statist. Phys. 7 (1973), 295-300.

20. D. H. Wagner, Survey of measurable selection theorems, SIAM J. Control Optim. 15 (1977), 859-903.

21. E. Wesley, Borel preference orders in markets with a continuum of traders, J. Math. Econom. 3 (1976), $155-165$.

22. A. Wilansky, Modern methods in topological vector spaces, McGraw-Hill, New York, 1978.

Department of Economics, Cornell University, Ithaca, New York 14853

Department of Political Economy, Johns Hopkins University, Baltimore, Maryland 21218

Current address: Department of Economics, University of Illinois, 1206 South Sixth Street, Champaign, Illinois 61820 